\title{
miR-142-3p promotes osteoblast differentiation by modulating Wnt signaling
}

\author{
WEIHUA HU, YAPING YE, WEIKAI ZHANG, JIANG WANG, ANMIN CHEN and FENGJING GUO \\ Department of Orthopedics, Tongji Hospital, Tongji Medical College, \\ Huazhong University of Science and Technology, Wuhan 430030, P.R. China
}

Received August 26, 2012; Accepted November 22, 2012

DOI: $10.3892 / \mathrm{mmr} .2012 .1207$

\begin{abstract}
Canonical Wnt signaling is critical for the control of osteoblast differentiation in human mesenchymal stem cells. MicroRNAs (miRs) are essential regulators of cell differentiation by post-transcriptional regulation of target gene expression. The aim of the present study was to investigate the molecular mechanism by which miR-142-3p promotes osteoblastic differentiation using the human fetal osteoblastic 1.19 (hFOB1.19), real-time PCR and western blot analysis. Results showed an increased expression of miR-142-3p during osteoblast differentiation in the mesenchymal precursor cell line, hFOB1.19. In addition, the ectopic overexpression of miR-142-3p promoted hFOB1.19 differentiation, whereas the inhibition of miR-142-3p repressed differentiation. The expression of miR-142-3p was positively correlated with $\beta$-catenin, an important protein in Wnt signaling. The adenomatous polyposis coli (APC) gene was a direct target of miR-142-3p, whereby miR-142-3p promoted Wnt signaling through inhibition of APC, leading to accumulation and nuclear translocation of $\beta$-catenin. Therefore, miR-142-3p may be an essential mediator of osteoblast differentiation and a new therapeutic strategy for osteogenesis disorders.
\end{abstract}

\section{Introduction}

Mesenchymal stem cells (MSCs) differentiate along several lineages, including osteoblasts, adipocytes and myoblasts, in response to various environmental stimuli (1). MSC differentiation is tightly regulated at transcriptional and

Correspondence to: Dr Fengjing Guo, Department of Orthopedics, Tongji Hospital, Tongji Medical College, Huazhong University of Science and Technology, 1095 Jiefang Avenue, Wuhan 430030, P.R. China

E-mail: tjfj_guo@163.com

Abbreviations: MSCs, mesenchymal stem cells; Runx2, runt-related transcription factor 2; APC, adenomatous polyposis coli; GSK3 $\beta$, glycogen synthase kinase $3 \beta$; CKI, casein kinase $1 \mathrm{a}$; hFOB, human fetal osteoblastic; OC, osteocalcin; ALP, alkaline phosphatase

Key words: miR-142-3p, Wnt, APC, osteoblast, differentiation post-transcriptional levels (2). A number of genes are important for the regulation of MSC differentiation, including bone morphogenetic protein and runt-related transcription factor 2 (Runx2) $(3,4)$. Canonical Wnt signaling is also critical for the control of bone formation (5) as it accelerates the differentiation of osteochondral progenitors towards the osteoblastic lineage, while promoting early MSC proliferation (6). In addition, Wnt signaling functions as an adipogenic switch, whereby loss of Wnt/ $\beta$-catenin signaling drives the shift of preosteoblasts from osteoblast to adipocyte differentiation (5).

The canonical Wnt pathway requires binding of Wnt to low-density lipoprotein receptor-related proteins 5 and 6 , Frizzled receptor and Dishevelled. In the absence of Wnt stimulation, cytoplasmic $\beta$-catenin is recruited by a multiprotein destruction complex consisting of two scaffold proteins, adenomatous polyposis coli (APC) and axin and two kinases, glycogen synthase kinase $3 \beta$ (GSK3 $\beta$ ) and casein kinase 1a (CKI). This complex leads to constitutive phosphorylation of $\beta$-catenin and its subsequent proteasomal degradation. Wnt receptor binding and activation inhibits GSK3 $\beta$ activity, leading to accumulation and nuclear translocation of $\beta$-catenin, which functions as a transcriptional coactivator and drives the transcription of genes essential for osteoblast differentiation. Numerous factors regulate osteoblast differentiation through the Wnt-mediated pathway (7).

MicroRNAs (miRNAs/miRs) negatively regulate translation or induce degradation of target mRNAs by binding to their 3' untranslated regions (UTRs). miRNAs are potent regulators of diverse physiological processes, including cell proliferation, differentiation and apoptosis (8). It was recently reported that miRNAs are essential for the regulation of osteoblastic differentiation (2). Huang et al demonstrated that miR-204 inhibits osteoblast differentiation by targeting Runx2 in bone marrow-derived MSC (4). miR-138 represses osteoblast differentiation by inhibiting FAK (9). By contrast, miR-29b promotes osteoblast differentiation by targeting inhibitors of this process (10). A number of miRNAs regulate osteoblast differentiation through the Wnt signaling pathway. miR-29a potentiates Wnt signaling and regulates osteoblast differentiation (6). miR-27 positively regulates osteoblast differentiation by targeting APC (11). miR-142-3p regulates myeloid and erythroid differentiation $(12,13)$, however, the role of miR-142-3p in regulating osteoblast differentiation remains unknown.

In the present study, the molecular mechanism by which miR-142-3p promotes osteoblastic differentiation 
was investigated. Results showed that miR-142-3p activated Wnt signaling and promoted osteoblast differentiation by repressing APC expression.

\section{Materials and methods}

Cell culture. The human fetal osteoblastic 1.19 (hFOB1.19) cell line was obtained from the American Type Culture Collection (Manassas, VA, USA) and cultured at $33.5^{\circ} \mathrm{C}$ in medium composed of 1:1 Dulbecco's modified Eagle's medium/Ham's F-12 medium without phenol red, supplemented with $10 \%$ FBS (Atlas Biologicals, Fort Collins, CO, USA), 1X penicillin/ streptomycin (Invitrogen Life Technologies, Carlsbad, CA, USA) and $0.3 \mathrm{mg} / \mathrm{ml} \mathrm{G} 418 /$ geneticin (Calbiochem, La Jolla, CA, USA). To induce osteoblastic differentiation, confluent cultures of hFOB1.19 cells were maintained in complete medium containing the following differentiation cocktail: $100 \mu \mathrm{g} / \mu \mathrm{l}$ ascorbic acid, $5 \mathrm{mM} \beta$-glycerol phosphate, $10^{-8} \mathrm{M}$ menadione and $10^{-7} \mathrm{M} 1,25(\mathrm{OH}) 2 \mathrm{D} 3$ (all from Sigma, St. Louis, MO, USA).

Plasmid constructs. Sequences containing the predicted binding sites in the $3^{\prime}$ UTR of APC mRNA were amplified and cloned into the Dual-Luciferase reporter vector, psiCHECK2 (Promega Corporation, Madison, WI, USA). Binding site mutations were introduced by whole-plasmid amplification in the seed region of miR-142-3p (New England Biolabs, Ipswich, MA, USA). Pre-miR-142 and anti-miR-142 were obtained from GeneCopoeia (Rockville, MD, USA).

Luciferase activity assay. HEK 293 cells were plated in 96-well plates and transfected with $0.02 \mu \mathrm{g}$ wild-type or mutated psiCHECK2-APC 3' UTR, with $0.08 \mu \mathrm{g}$ pre-miR-142 (MI0000167) or vector (GeneCopoeia). Each transfection was repeated twice in triplicate. Following $48 \mathrm{~h}$ of transfection, firefly and Renilla luciferase activities were measured with the Dual-Luciferase ${ }^{\circledR}$ Reporter 1000 Assay System (Promega Corporation).

Real-time quantitative PCR. Total RNA was extracted with TRIzol (Invitrogen Life Technologies). cDNA was synthesized using $1 \mu \mathrm{g}$ RNA with a reverse transcription kit (Tiangen Biotech, Beijing, China). miRNAs and the synthesized cDNAs were prepared with a microRNA extraction kit (Tiangen Biotech). Real-time PCR was performed with a standard SYBR-Green PCR kit from Toyobo (Osaka, Japan). Primers used included: $\beta$-actin F: 5'-AGCCATGTACGTTGCTA-3', R: 5'-AGTCCGCCTAGAAGCA-3'; Runx2 F: 5'-TCTTCACA AATCCTCCCC-3', R: 5'-TGGATTAAAAGGACTTGG-3'; osteocalcin (OC) F: 5'-CATGAGAGCCCTCACA-3', R: 5'-AGA GCGACACCCTAGAC-3'; and alkaline phosphatase (ALP) F: 5'-ACGTGGCTAAGAATGTCATC-3', R: 5'-CTGGTAGGC GATGTCCTTA-3'. Primers for miR-142-3p and U6 snRNA were obtained from GeneCopoeia. Runx2, OC and ALP mRNA levels were normalized to $\beta$-actin and the expression of miR-142-3p was normalized to U6 snRNA.

Measurement of cell differentiation. Osteoblast marker transcripts (Runx2, OC and ALP) were detected by real-time PCR as described. ALP activity was measured as previously described (14).



Figure 1. Relative expression of miR-142-3p during osteoblastic differentiation. Confluent cultures of hFOB1.19 cells were maintained in complete medium and a specific differentiation cocktail. Cells were harvested following 12-, 48- and 72-h incubation and relative expression of miR-142-3p was measured. ${ }^{*} \mathrm{P}<0.05$ and ${ }^{* *} \mathrm{P}<0.01$, vs. non-differentiating group $(\mathrm{n}=3$ for all experiments).

Western blot analysis. Western blot analysis to measure $\mathrm{APC}$ and $\beta$-catenin protein levels was performed as previously described (15). Anti-APC and anti- $\beta$-catenin primary antibodies were purchased from Cell Signaling Technology (Beverly, MA, USA). Blots were incubated with anti-mouse or anti-rabbit IgG conjugated to IRDye ${ }^{\mathrm{TM}}(800 \mathrm{CW})$ for $1 \mathrm{~h}$ at $37^{\circ} \mathrm{C}$ and visualized using the Odyssey Infrared Imaging System (Licor Biosciences, Lincoln, NE, USA).

Statistical analysis. Data were expressed as the mean \pm SE. Data were analyzed using the Student's t-test. $\mathrm{P}<0.05$ was considered to indicate a statistically significant difference.

\section{Results}

Upregulated miR-142-3p promotes osteoblastic differentiation. Previous studies have demonstrated that miR-142-3p regulates myeloid and erythroid differentiation. We detected miR-142-3p expression during hFOB1.19 cell differentiation. miR-142-3p increased in hFOB1.19 cells maintained in osteoblastic differentiation medium (Fig. 1).

To investigate the potential function of miR-142-3p in hFOB1.19 differentiation, cells were transfected with control vector, pre-miR-142 or anti-miR-124. Pre-miR-142 enhanced osteoblastic differentiation, which was indicated by higher expression of the osteoblast marker genes Runx2, OC and ALP (16), accompanied by increased ALP activity in comparison to cells transfected with control vector. By contrast, ALP activity and osteoblast-specific genes were identified to be significantly decreased when hFOB1.19 cells were transfected with anti-miR-142, a blocker of miR-142 (Fig. 2). These data indicate that upregulation of miR-142-3p positively regulated hFOB1.19 differentiation, whereas inhibition of miR-142-3p negatively regulated $\mathrm{hFOB} 1.19$ differentiation.

miR-142-3p targets APC via predicted binding sites. Prediction Algorithms miRbase and Targetscan were used to select potential targets of miR-142-3p in osteoblastic differentiation. miR-142-3p targets APC, which acts as a regulatory factor in Wnt signaling (Fig. 3A). In addition, luciferase reporter constructs containing possible binding sites and specific mutations within 
A



B

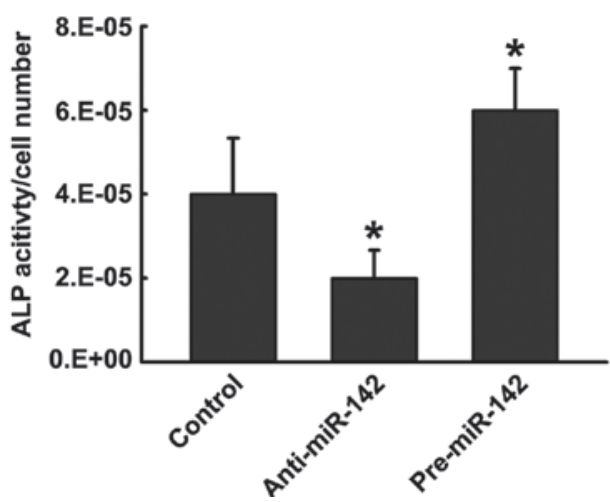

Figure 2. miR-142-3p promotes osteoblastic differentiation of hFOB1.19 cells. Cells were transiently transfected with pre-miR-142, anti-miR-142 or control vector for 3 days under osteoblast differentiation conditions. (A) Osteoblast marker transcript levels (Runx2, OC and ALP) were evaluated. (B) Alkaline phosphatase activity was measured. $\mathrm{P}<0.05$ and ${ }^{* *} \mathrm{P}<0.01$, vs. control ( $\mathrm{n}=3$ for all experiments). miR, microRNA; $\mathrm{hFOB}$, human fetal osteoblastic; Runx2, runt-related transcription factor 2; OC, osteocalcin; ALP, alkaline phosphatase.

A

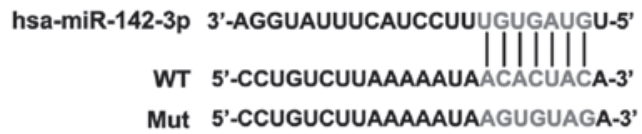

B

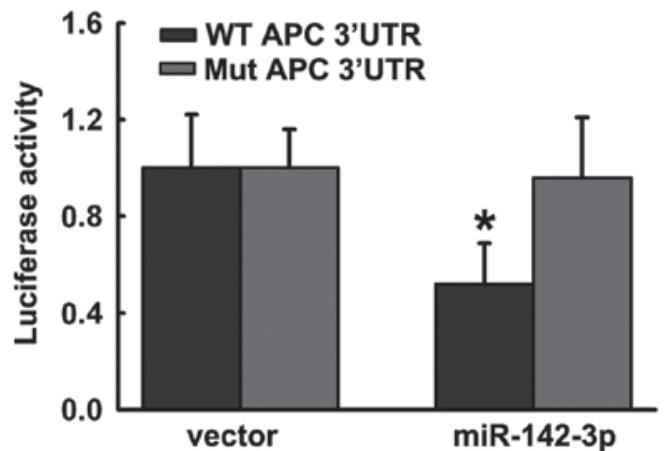

C
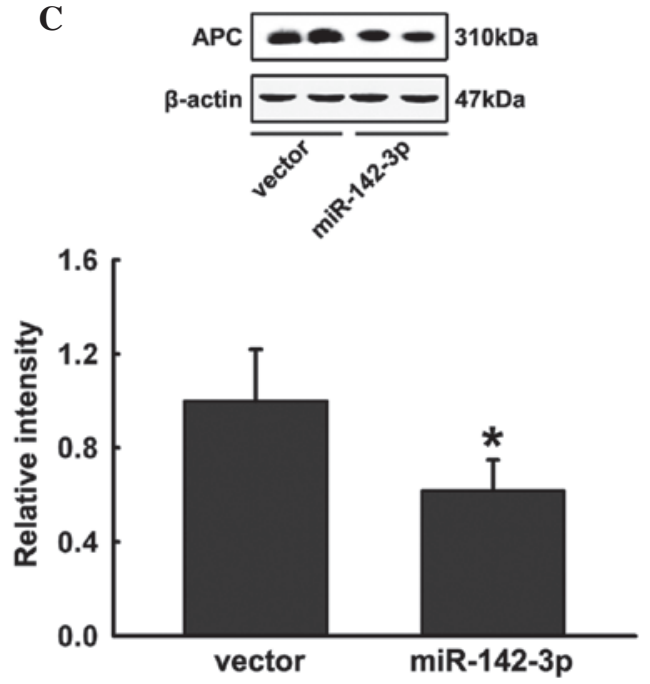

Figure 3. miR-142-3p targets APC via predicted binding sites. (A) Schematic diagram of WT and Mut APC 3' UTR luciferase constructs. (B) Dual luciferase assays in HEK293 cells. Reporter constructs containing WT or Mut APC 3' UTR were cotransfected with the miR-142-3p overexpression plasmid or control vector. Relative luciferase activity was normalized to firefly luciferase. (C) Western blot analysis of APC protein levels following transfection of the miR-142-3p overexpression plasmid. Data are representative of 3 separate experiments (Student's t-test; ${ }^{~} \mathrm{P}<0.05$, vs. vector). miR, microRNA; APC, adenomatous polyposis coli; UTR, untranslated region; WT, wild-type; Mut, mutant.

A

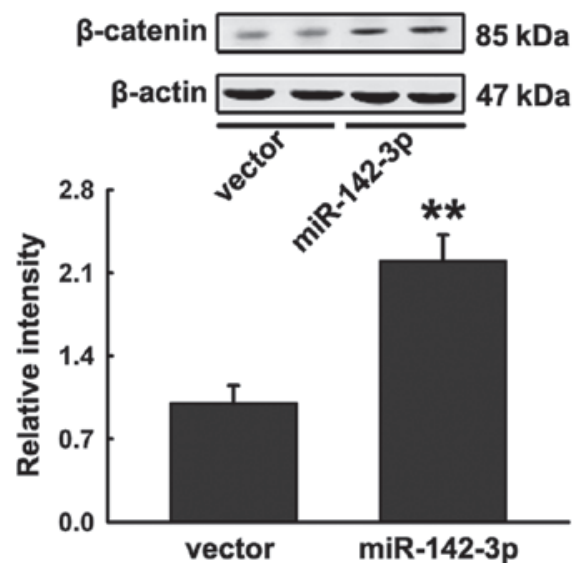

B

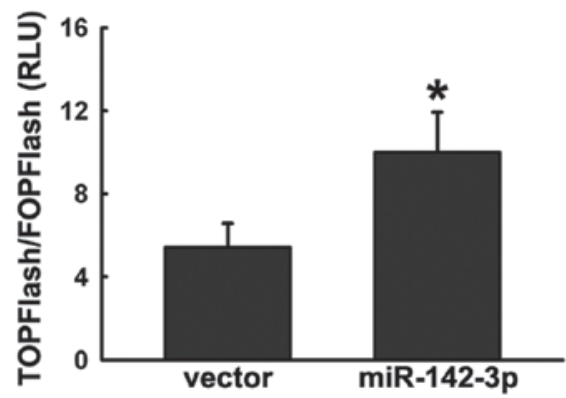

Figure 4. miR-142-3p promotes Wnt signaling. (A) Western blot analysis of $\beta$-catenin in hFOB1.19 cells transfected with miR-142-3p overexpression plasmid or control vector. (B) Luciferase activity of TOPFlash/FOPFlash in hFOB1.19 cells transfected with miR-142-3p overexpression plasmid or the control vector. ${ }^{*} \mathrm{P}<0.05$ and ${ }^{* *} \mathrm{P}<0.01$, vs. vector ( $\mathrm{n}=3$ for all experiments). miR, microRNA; hFOB, human fetal osteoblastic. 
the 3' UTR of APC mRNA were generated. Wild-type and mutated APC 3' UTR luciferase reporter constructs were cotransfected into HEK293 cells with the miR-142-3p overexpression plasmid or a control vector. Significant inhibition was observed when miR-142-3p was cotransfected with the wild-type APC 3' UTR in HEK293 cells, whereas mutated APC 3' UTR luciferase activity was altered marginally in comparison to the vector control (Fig. 3B). The reporter assay demonstrated that miR-142-3p significantly repressed luciferase expression and mutation of four nucleotides in the binding sites led to complete abrogation of this suppressive effect. Moreover, the ectopic expression of miR-142-3p significantly reduced APC protein levels (Fig. 3C). These observations indicate that miR-142-3p may inhibit endogenous APC expression in differentiating hFOB1.19 cells.

miR-142-3p promotes Wnt signaling by APC inhibition. Previous studies have revealed that APC negatively regulates Wnt signaling by interacting with $\beta$-catenin, a key component of the pathway (17). Ectopic expression of miR-142-3p in hFOB1.19 cells significantly increased accumulation of $\beta$-catenin (Fig. 4A). To confirm the effect on Wnt signaling, hFOB1.19 cells were cotransfected with pre-miR-142 or miRNA control with the Wnt signaling reporter TOPFlash or negative control FOPFlash and then treated with Wnt3a. Cells transfected with pre-miR-142 were observed to significantly increase TOPFlash activity (Fig. 4B). These observations indicate that miR-142-3p positively regulates hFOB1.19 differentiation by activating Wnt signaling.

\section{Discussion}

miRNAs are small non-coding RNA molecules that post-transcriptionally regulate gene expression by targeting sequences within the 3' UTR of target mRNAs. miRNAs are involved in several essential biological processes, including tumorigenesis, hormone signaling and differentiation (8) and are critical for the control of adipogenesis, myeloblast differentiation and osteoblastogenesis (2). In the present study, miR-142-3p was identified to positively regulate osteoblastic differentiation by promoting Wnt signaling.

miR-142-3p is specifically associated with the regulation of hematopoietic lineage specification, obesity and periodontitis by targeting diverse molecules, including glucocorticoid receptor- $\alpha$ and IL-6 (18,19). Kim et al reported that miR-142-3p is involved in TGF- $\beta 3$-mediated region-dependent chondrogenesis through regulation of ADAM9 (20). miR-142-3p is an essential regulator of myeloid differentiation and downregulation of miR-142-3p is involved in the development of acute myeloid leukemia $(12,18)$. miR-142-3p is also involved in erythroid differentiation of human embryonic stem cells (13). However, the potential function of miR-142-3p in the regulation of osteoblastic differentiation remains unclear. Our results showed that miR-142-3p levels increased in differentiating hFOB1.19 cells and overexpression promoted differentiation. When miR-142-3p function was inhibited, Runx2, OC and ALP mRNA levels were significantly reduced, accompanied by decreased ALP activity. In addition, miR-142-3p was observed to target APC, an important Wnt signaling molecule. The luciferase reporter assay revealed that miR-142-3p significantly inhibited APC
3' UTR expression. Western blot analysis also confirmed that miR-142-3p significantly suppressed APC protein levels in differentiating hFOB1.19 cells.

APC is a scaffold protein for GSK3 $\beta / \mathrm{CKI}$-mediated phosphorylation and subsequent degradation of $\beta$-catenin, the Wnt signaling effector. Without Wnt stimulation, $\beta$-catenin interacts with a multiprotein complex of APC, axin, GSK $3 \beta$ and CKI, known as the destruction complex, leading to phosphorylation of $\beta$-catenin by GSK3 $\beta$ and CKI. Once phosphorylated, $\beta$-catenin is ubiquitinated and degraded by the proteasome. Activation of Wnt signaling may inhibit GSK3 $\beta$ activity and result in the accumulation of non-phosphorylated $\beta$-catenin in the cytoplasm and nuclear translocation. Binding of the Tcf/Lef family of transcription factors promotes target gene expression (17). Blocking $\beta$-catenin binding sites on APC inhibits $\beta$-catenin degradation and leads to unchecked Wnt signaling (21). Therefore, APC inhibition by miR-142-3p may disrupt the destruction complex, induce nuclear accumulation of $\beta$-catenin and activate Wnt signaling.

The present study has demonstrated that miR-142-3p positively regulates osteoblast differentiation by targeting APC, indicating that miR-142-3p may be an important candidate therapeutic agent for treatment of osteogenesis disorders.

\section{References}

1. Kang SK, Shin IS, Ko MS, Jo JY and Ra JC: Journey of mesenchymal stem cells for homing: strategies to enhance efficacy and safety of stem cell therapy. Stem Cells Int 2012: 342968, 2012.

2. Lian JB, Stein GS, van Wijnen AJ, et al: MicroRNA control of bone formation and homeostasis. Nat Rev Endocrinol 8: 212-227, 2012.

3. Suzuki D, Yamada A, Aizawa R, et al: BMP2 differentially regulates the expression of Gremlin1 and Gremlin2, the negative regulators of BMP function, during osteoblast differentiation. Calcif Tissue Int 91: 88-96, 2012.

4. Huang J, Zhao L, Xing L and Chen D: MicroRNA-204 regulates Runx 2 protein expression and mesenchymal progenitor cell differentiation. Stem Cells 28: 357-364, 2009.

5. Song L, Liu M, Ono N, Bringhurst FR, Kronenberg HM and Guo J: Loss of wnt/ $\beta$-catenin signaling causes cell fate shift of preosteoblasts from osteoblasts to adipocytes. J Bone Miner Res: Jun. 22, 2012 (Epub ahead of print).

6. Kapinas K, Kessler C, Ricks T, Gronowicz G and Delany AM: miR-29 modulates Wnt signaling in human osteoblasts through a positive feedback loop. J Biol Chem 285: 25221-25231, 2010.

7. Herr P, Hausmann G and Basler K: WNT secretion and signalling in human disease. Trends Mol Med 18: 483-493, 2012.

8. Pritchard CC, Cheng HH and Tewari M: MicroRNA profiling: approaches and considerations. Nat Rev Genet 13: 358-369, 2012.

9. Eskildsen T, Taipaleenmaki H, Stenvang J, et al: MicroRNA-138 regulates osteogenic differentiation of human stromal (mesenchymal) stem cells in vivo. Proc Natl Acad Sci USA 108: 6139-6144, 2011

10. Li Z, Hassan MQ, Jafferji M, et al: Biological functions of miR-29b contribute to positive regulation of osteoblast differentiation. J Biol Chem 284: 15676-15684, 2009.

11. Wang T and Xu Z: miR-27 promotes osteoblast differentiation by modulating Wnt signaling. Biochem Biophys Res Commun 402: 186-189, 2010.

12. Wang XS, Gong JN, Yu J, et al: MicroRNA-29a and microRNA$142-3 p$ are regulators of myeloid differentiation and acute myeloid leukemia. Blood 119: 4992-5004, 2012.

13. Jin HL, Kim JS, Kim YJ, Kim SJ, Broxmeyer HE and Kim KS: Dynamic expression of specific miRNAs during erythroid differentiation of human embryonic stem cells. Mol Cells 34: 177-183, 2012.

14. Qiu W, Hu Y, Andersen TE, et al: Tumor necrosis factor receptor superfamily member 19 (TNFRSF19) regulates differentiation fate of human mesenchymal (stromal) stem cells through canonical Wnt signaling and C/EBP. J Biol Chem 285: 14438-14449, 2010. 
15. Zhu HC, Wang LM, Wang M, et al: MicroRNA-195 downregulates Alzheimer's disease amyloid- $\beta$ production by targeting BACE1. Brain Res Bull 88: 596-601, 2012.

16. Abdallah BM, Haack-Sorensen M, Burns JS, et al: Maintenance of differentiation potential of human bone marrow mesenchyma stem cells immortalized by human telomerase reverse transcriptase gene despite [corrected] extensive proliferation. Biochem Biophys Res Commun 326: 527-538, 2005.

17. Akiyama T: Wnt/beta-catenin signaling. Cytokine Growth Factor Rev 11: 273-282, 2000.

18. Wang F, Wang XS, Yang GH, et al: miR-29a and miR-142-3p downregulation and diagnostic implication in human acute myeloid leukemia. Mol Biol Rep 39: 2713-2722, 2011
19. Perri R, Nares S, Zhang S, Barros SP and Offenbacher S: MicroRNA modulation in obesity and periodontitis. J Dent Res 91: 33-38, 2011.

20. Kim D, Song J, Kim S, Kang SS and Jin EJ: MicroRNA-142-3p regulates TGF- $\beta 3$-mediated region-dependent chondrogenesis by regulating ADAM9. Biochem Biophys Res Commun 414: 653-659, 2011.

21. Waaler J, Machon O, Tumova L, et al: A novel tankyrase inhibitor decreases canonical Wnt signaling in colon carcinoma cells and reduces tumor growth in conditional APC mutant mice. Cancer Res 72: 2822-2832, 2012. 